\title{
Uso da Angiografia Rotacional 3D (3D-RA) em Portadores de Cardiopatias Congênitas: Experiência de 53 Casos
}

\author{
Carlos Eduardo Bernini Kapins ${ }^{1}$, Ralph Bittencourt Coutinho' ${ }^{1}$, Fabiane Berruezo Barbosa', \\ Célia Maria Camelo Silva', Valter Correa Lima', Antonio Carlos Carvalho'
}

\section{RESUMO}

Introdução: Os avanços no diagnóstico e tratamento das cardiopatias congênitas estão relacionados com os avanços na obtenção de imagens. As representações anatômicas obtidas com tomografia computadorizada, ressonância magnética e ecocardiografia têm ajudado, mas esses métodos não conseguem obter de forma precisa os dados hemodinâmicos. A angiografia rotacional 3D (3D-RA), um novo método de reconstrução tridimensional realizado no laboratório de cateterismo, tem sido amplamente utilizada em procedimentos neurológicos e urológicos. Nosso objetivo foi avaliar o desempenho da 3D-RA na avaliação das cardiopatias congênitas. Método: Revisão dos exames dos pacientes com cardiopatia congênita encaminhados para estudo hemodinâmico e que foram submetidos a aquisição de imagens pela 3D-RA. Foi utilizado equipamento Philips Allure FD10 e contraste de baixa osmolaridade para as angiografias. Resultados: No total, foram avaliados 53 pacientes, sendo utilizados, em média, 2,2 $\pm 1,1 \mathrm{ml} / \mathrm{kg}$ de contraste por paciente. Foi possível observar detalhes anatômicos não revelados em angiografias planares em $23 \%$ dos pacientes. Adicionalmente, $49 \%$ dos pacientes tiveram benefícios das imagens 3D para decisões terapêuticas. A exposição à radiação não foi estatisticamente diferente das angiografias planares. Nenhum paciente apresentou complicações relacionadas ao método. Conclusão: A 3D-RA forneceu informações que algumas das angiografias convencionais não fornecem e que ajudaram no tratamento de pacientes selecionados portadores de cardiopatia congênita. O emprego desse método poderá reduzir o número de aquisições de imagem por procedimento e, como consequência, limitar a exposição do paciente à radiação e ao contraste.

DESCRITORES: Cardiopatias congênitas. Angiografia. Exposição a radiação.

\footnotetext{
1 Universidade Federal de São Paulo - Escola Paulista de Medicina - São Paulo, SP, Brasil.

Correspondência: Carlos Eduardo Bernini Kapins. Rua Botucatu, 740

- Vila Clementino - São Paulo, SP, Brasil - CEP 04023-900

E-mail: carlosbernini@me.com

Recebido em: 5/4/2010 • Aceito em: 1\%/6/2010
}

\author{
ABSTRACT \\ Use of 3D Rotational Angiography (3D-RA) \\ in Congenital Heart Disease: Experience \\ with 53 Patients
}

Background: Advances in the diagnosis and treatment of congenital heart disease are associated to advances in imaging techniques. Anatomic images obtained by computed tomography scan, magnetic resonance imaging (MRI) and echocardiography have been useful but cannot provide accurate hemodynamic data. 3D rotational angiography (3D-RA) is a new 3D reconstruction method carried out in the cath lab that has been widely used in neurological and urological procedures. This study was aimed at evaluating the use of 3D-RA in the diagnosis and treatment of congenital heart disease. Method: Review of catheterization results of patients with congenital heart disease referred for diagnostic assessment in which the $3 \mathrm{D}$ reconstruction method was employed. Philips Allure FD 10 equipment and low osmolarity contrast medium were used for angiographies. Results: Overall, 53 patients were reviewed and $2.2 \pm 1.1 \mathrm{~mL} / \mathrm{kg}$ of contrast medium were used per patient. Anatomic details not previously shown by 2D angiographies were observed in $23 \%$ of the patients. Furthermore, 3D-RA imaging was used to make treatment decisions in $49 \%$ of the patients. Exposure to radiation was not statistically different from $2 \mathrm{D}$ angiography. None of the patients had complications related to the method. Conclusion: 3D-RA provided information not usually seen by conventional angiography which was useful in the treatment of selected patients with congenital heart disease. The use of 3D-RA may reduce the number of imaging tests per procedure and as a consequence, limit patient exposure to radiation and contrast media.

KEY-WORDS: Heart defects, congenital. Angiography. Radiation exposure. 
que, nos últimos 30 anos, tiveram grande salto de tecnologia e precisão. As representações tridimensionais são as representações anatômicas que, na última década, ganharam grande espaço no diagnóstico e no tratamento desses pacientes. Os métodos capazes de realizar as imagens tridimensionais são a ressonância magnética, a tomografia computadorizada, a ecografia e, mais recentemente, a angiografia rotacional. Entretanto, como somente a angiografia é realizada de forma invasiva, os demais métodos não podem medir pressões e nem calcular os coeficientes hemodinâmicos de forma precisa, somente de forma indireta.

Idealizada no final da década de 1960, a angiografia rotacional (3D-AR) surgiu apenas em meados da década de 1990 e tem sido de grande utilidade nas áreas de neurologia intervencionista, radiologia intervencionista e agora também nos estudos eletrofisiológicos. ${ }^{1-3}$

O uso da angiografia rotacional nas cardiopatias congênitas aparece em somente dois relatos de caso na literatura. Uma senhora de 62 anos que realizou oclusão percutânea do canal arterial e o segundo caso de uma criança de 1 ano com fístula coronária. ${ }^{4-6}$ Nosso objetivo foi avaliar o desempenho da 3D-AR na avaliação das cardiopatias congênitas.

\section{MÉTODO}

De abril de 2008 a março de 2010 foram revisados os exames de todos os pacientes portadores de cardiopatia congênita encaminhados para realização de estudo hemodinâmico, diagnóstico ou terapêutico, no Setor de Hemodinâmica do Hospital São Paulo da Universidade Federal de São Paulo, nos quais foram feitas aquisições de imagens por 3D-AR, adicionalmente às imagens obtidas no exame planar convencional. Os critérios para a aquisição de imagens pela angiografia rotacional foram: necessidade de esclarecer o diagnóstico, necessidade de realizar medidas tridimensionais de estruturas de interesse para auxiliar a escolha do material apropriado para o procedimento intervencionista ou necessidade de obter melhor projeção angiográfica para a intervenção. Os dados disponíveis foram: idade, peso, altura, superfície corporal, diagnóstico da cardiopatia congênita e variáveis que expressam a radiação emitida e absorvida nas angiografias planar e 3D-RA. Essas variáveis, air kerma ${ }^{a}$ (AK - mGy) e dose-area product ${ }^{\text {b }}$ (DAP - mGy.cm²), foram obtidas para cada aquisição e as médias das imagens planar e rotacional foram comparadas em cada paciente.

\footnotetext{
a KERMA - Kinetic Energy Released per unit MAss (of air): medida da quantidade de radiação (em joules) depositada ou absorvida em uma unidade de massa $(\mathrm{kg})$ de ar. Expressa a concentração de radiação liberada em um ponto $(\mathrm{J} / \mathrm{kg}=\mathrm{Gy})$.

b Dose-Area Product: produto do air kerma, em Gy ou mGy, e a área exposta $\mathrm{em} \mathrm{cm}^{2}$. Fornece uma boa estimativa do total de radiação liberada para o paciente durante o procedimento.
}

Utilizamos o aparelho Philips Allure FD10 equipado com o ajuste vascular da angiografia rotacional adaptado para o tórax. Nessa regulagem o aparelho é capaz de rodar o eixo do braço em $\mathrm{C}$ em ângulo de 240 graus, adquirindo 120 imagens de 90 graus a -90 graus. Essas imagens são transferidas a uma workstation com o programa Philips Allure 3D-RA e após 100 segundos, aproximadamente, obtém-se a imagem tridimensional, que pode ser girada em todos os planos, em um ângulo de 360 graus, permitindo visão completa da estrutura analisada. O contraste utilizado em todos os pacientes foi o Hexabrix $320 \mathrm{mg} \mathrm{l} / \mathrm{ml}$, com ou sem diluição em soro fisiológico $(1: 2,2: 3)$. E a bomba de infusão utilizada foi a Medrad Mark $V$ plus.

Em todos os exames utilizamos fluoroscopia com 15 imagens por segundo e baixo contraste de imagem.

As comparações entre os grupos foram realizadas por meio do teste $t$ de Student pareado. A análise estatística foi realizada com o programa Numbers para Mac OS.

\section{RESULTADOS}

No período mencionado foram realizadas 62 aquisições de imagens tridimensionais em 53 procedimentos (1 3D-AR em 44 procedimentos e 2 3D-AR em 9 procedimentos). Os dados demográficos e o tipo de procedimento estão apresentados na Tabela 1. Foi utilizada uma média de $2,2 \pm 1,1 \mathrm{ml} / \mathrm{kg}$ de contraste radiológico. Foram usados maiores volumes no início da experiência do Serviço e nos casos com grandes curtos-circuitos (shunts) entre cavidades esquerdas e direitas.

A análise subjetiva das imagens obtidas por meio do 3D-AR classificou a qualidade das aquisições como excelente em 45\% dos casos, boa em 39\% e não-aproveitável em 16\%, e a maioria das não-aproveitáveis foi adquirida no começo da experiência. Cerca de metade dos pacientes $(38 \%$ dos procedimentos intervencionistas e $11 \%$ dos procedimentos cirúrgicos) se beneficiaram

\section{TABELA 1 \\ Características demográficas e tipos de procedimento}

\begin{tabular}{lc}
\hline Masculino, $\mathrm{n}$ & 28 \\
Feminino, $\mathrm{n}$ & 25 \\
Procedimento diagnóstico, $\mathrm{n}$ & 33 \\
Procedimento terapêutico, $\mathrm{n}$ & 20 \\
Idade*, anos $^{*}$ & 6 \\
Peso $^{* *}, \mathrm{~kg}$ & $28,5 \pm 18,6$ \\
Altura**, cm & $124,4 \pm 31,4$ \\
Superfície corporal**, $\mathrm{m}^{2}$ & $0,97 \pm 0,43$ \\
\hline$*$ Mediana. & \\
$* *$ Média \pm desvio padrão. & \\
$\mathrm{n}=$ número de pacientes. & \\
\hline
\end{tabular}


Kapins CEB, et al. Uso da Angiografia Rotacional 3D (3D-RA) em Portadores de Cardiopatias Congênitas: Experiência de 53 Casos. Rev Bras Cardiol Invasiva. 2010;18(2):199-203.

das imagens tridimensionais angiográficas, seja para esclarecimento diagnóstico seja para guiar a escolha da prótese ou fornecer a melhor incidência para a realização do procedimento. Em 23\% dos casos a 3D-AR acrescentou informações anatômicas adicionais à angiografia planar.

A produção de radiação (AK) foi maior com o uso da 3D-AR que com a angiografia planar, mas o aumento não foi estatisticamente significante $(P=0,054)$. A diferença de quantidade de radiação absorvida (DAP) não mostrou diferença entre os grupos (Tabela 2).

Na Figura 1 observa-se a distribuição dos tipos de afecções estudadas pela 3D-RA.

Não observamos nenhuma complicação relacionada à execução da angiografia rotacional e a quantidade total de contraste angiográfico utilizado permaneceu dentro dos limites preconizados. Nas Figuras 2 a 5 observam-se exemplos da angiografia tridimensional.

TABELA 2

Comparação das variáveis que expressam a radiação emitida e absorvida por aquisição de imagem nas angiografias planar e 3D-RA

\begin{tabular}{lccc}
\hline & $\begin{array}{c}\text { Angiografia } \\
\text { planar }\end{array}$ & $\begin{array}{c}\text { Angiografia } \\
\text { 3D-RA }\end{array}$ & P \\
\hline $\mathrm{AK}(\mathrm{mGy})$ & $30.019 \pm 27.516$ & $41.467 \pm 27.561$ & 0,054 \\
$\mathrm{DAP}\left(\mathrm{mGy} . \mathrm{cm}^{2}\right)$ & $3.565 \pm 3.274$ & $3.745 \pm 2.281$ & 0,545 \\
\hline $\mathrm{AK}=$ air kerma; $\mathrm{DAP}=$ dose-area product. & \\
\hline
\end{tabular}

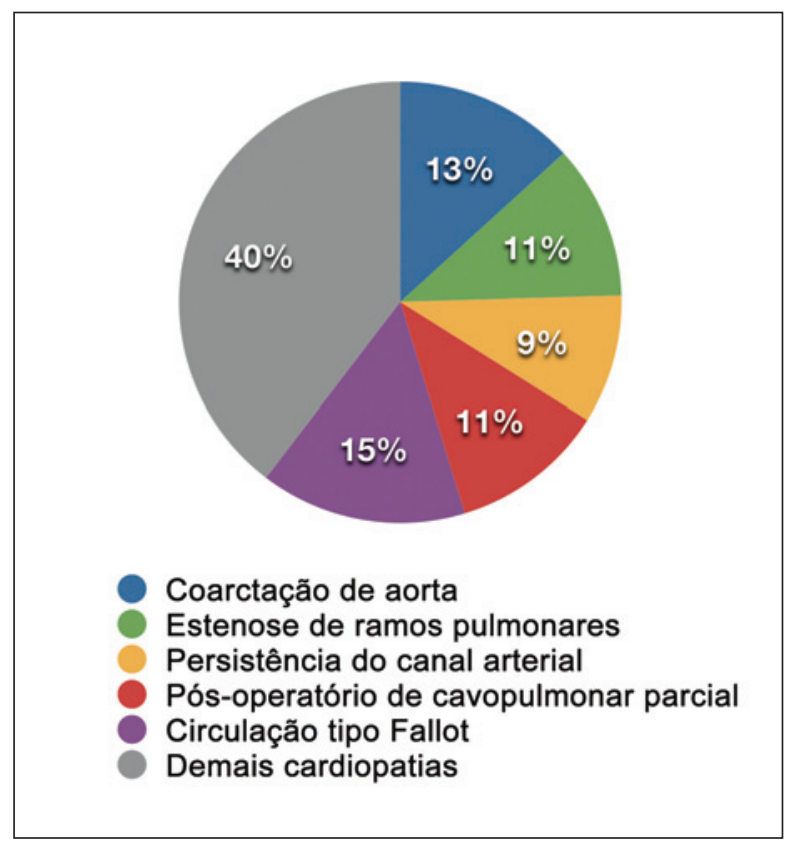

Figura 1 - Distribuição dos tipos de cardiopatias congênitas estudadas.

\section{DISCUSSÃO}

A angiografia planar tradicional é limitada pela aquisição de imagens bidimensionais de estruturas anatômicas complexas, como as habitualmente encontra-

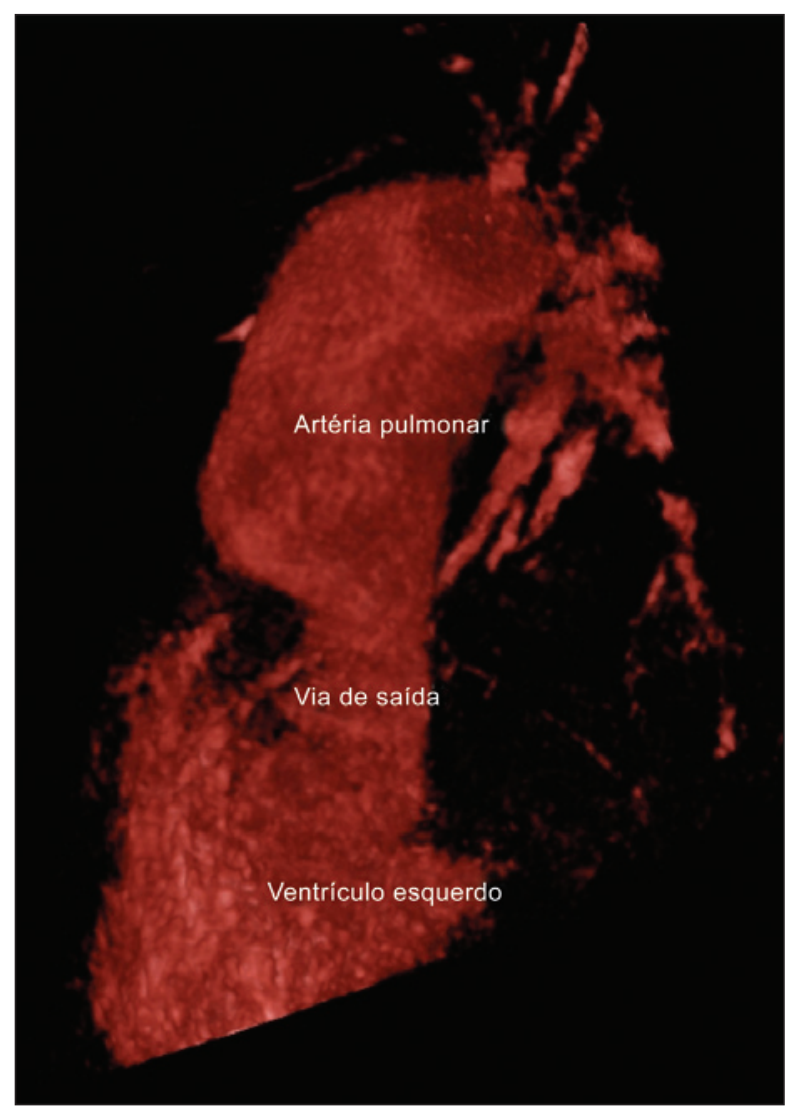

Figura 2 - Ventriculografia esquerda de um paciente com transposição corrigida dos grandes vasos. Nota-se a estenose subpulmonar, bem evidenciada em uma única angiografia.

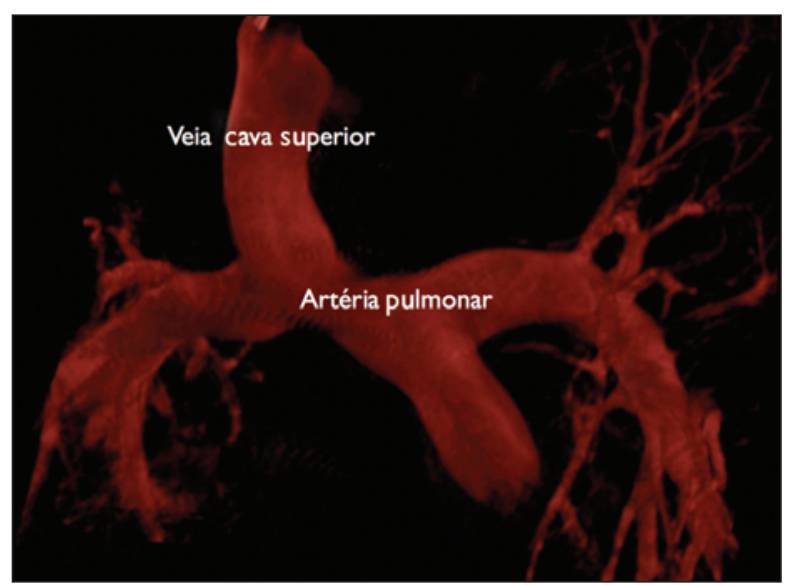

Figura 3 - Pós-operatório de derivação cavopulmonar parcial em um paciente com atresia tricúspide em programação para cavopulmonar total 


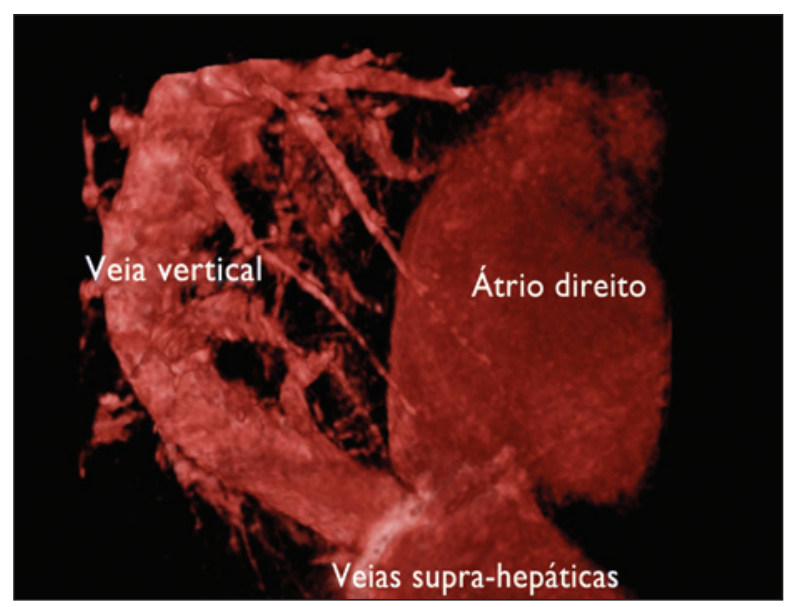

Figura 4 - Síndrome da cimitarra.

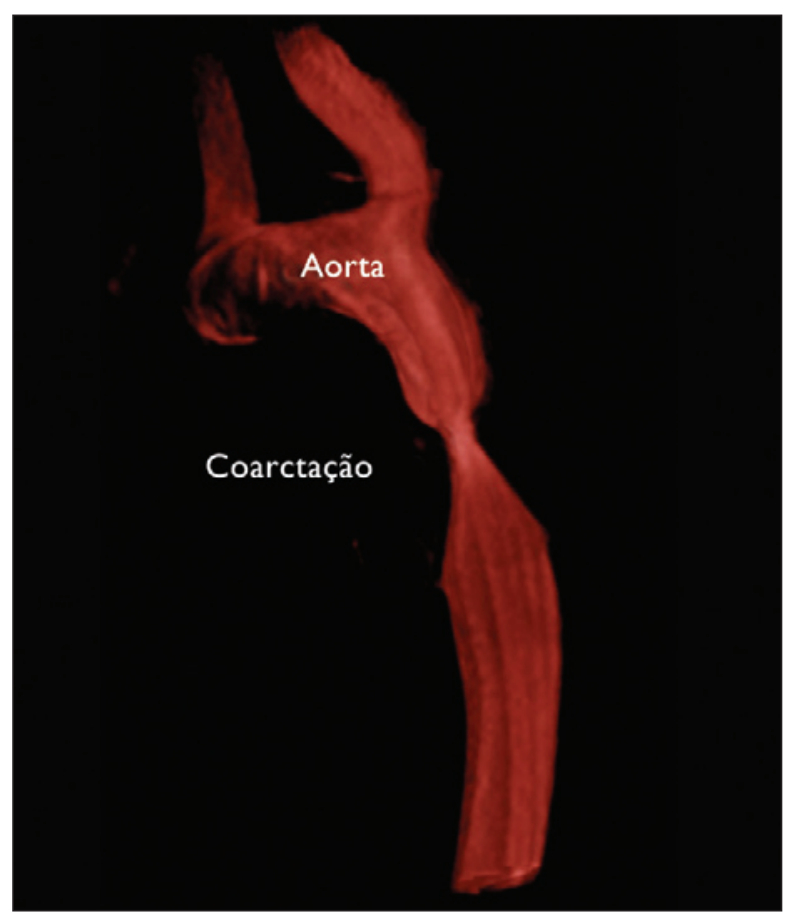

Figura 5 - Coarctação de aorta.

das nas cardiopatias congênitas. A angiografia rotacional e a reconstrução das imagens em 3D são duas novas ferramentas no diagnóstico e tratamento dessas cardiopatias e representam importante avanço na área de imagens, fornecendo uma multiplicidade de projeções da árvore vascular, com uma única injeção de contraste e riqueza de detalhes que a técnica planar não fornece. A literatura disponível a respeito de sua aplicação na avaliação das cardiopatias congênitas, no entanto, é escassa.

A curva de aprendizado do método é relativamente curta, mas existe a necessidade de treinamento da equipe e conhecimento das etapas do procedimento, para que não ocorra colisão do aparelho com acessos venosos, eletrodos de monitorização ou equipamentos de ventilação artificial durante a angiografia.

A qualidade das imagens vasculares obtidas é equivalente à da ressonância magnética e da tomografia computadorizada; assim, em nossa opinião, nos casos que necessitem da obtenção de pressões intracavitárias ou em que se planeja o tratamento com intervenção percutânea, apenas a 3D-RA poderia ser realizada, reduzindo o número de exames a que o paciente se submete. Uma limitação atual do método está na realização de ventriculografias, que, de modo geral, não mostram boa imagem 3D por causa do fenômeno de aliasinga , decorrente da contração miocárdica durante o ciclo cardíaco.

A 3D-RA pode ser de grande valia na escolha de próteses e balões para intervenções percutâneas, e, em alguns casos, pode mostrar a melhor projeção para realizar o procedimento, diminuindo, dessa maneira, o tempo de fluoroscopia, com consequente redução da exposição do paciente à radiação e ao contraste.

Nossos dados demonstraram que a utilização da 3D-AR forneceu informações adicionais, que não são obtidas nas angiografias planares, e que nos ajudaram a diagnosticar e a tratar pacientes com anatomia complexa. Novos estudos são necessários para estabelecer com precisão os nichos de indicação dessa técnica promissora, que fornece reconstruções tridimensionais de alta qualidade das estruturas anatômicas cardiovasculares.

\section{CONCLUSÃO}

A 3D-RA traz informações adicionais às obtidas com a angiografia planar e que ajudaram no diagnóstico e tratamento de pacientes selecionados com cardiopatia congênita. $\mathrm{O}$ emprego desse método tem o potencial de reduzir o número de aquisições de imagem por procedimento e, como consequência, limitar a exposição do paciente à radiação e ao contraste.

\section{CONFLITO DE INTERESSES}

Os autores declararam inexistência de conflito de interesses relacionado a este manuscrito.

\section{REFERÊNCIAS}

1. Van Rooij WJ, Sprengers ME, Gast AN, Peluso JP, Sluzewski M. 3D rotational angiography: the new gold standard in the detection of additional intracranial aneurysms. AJNR Am J Neuroradiol. 2008;29(5):976-9.

2. Li JH, Haim M, Movassaghi B, Mendel JB, Chaudhry GM, Haffajee $\mathrm{Cl}$, et al. Segmentation and registration of three-

\footnotetext{
a Aliasing: distorção ou artefato que resulta quando o sinal reconstruído por amostragem é diferente do sinal original contínuo.
} 
Kapins CEB, et al. Uso da Angiografia Rotacional 3D (3D-RA) em Portadores de Cardiopatias Congênitas: Experiência de 53 Casos. Rev Bras Cardiol Invasiva. 2010;18(2):199-203.

dimensional rotational angiogram on live fluoroscopy to guide atrial fibrillation ablation: a new online imaging tool. Heart Rhythm. 2009;6(2):231-7.

3. Hagen G, Wadstrom J, Eriksson LG, Magnusson P, Magnussom M, Magnussom A. Three-dimensional rotational angiography of transplanted renal arteries: influence of an extended angle of rotation on beam-hardening artifacts. Acta Radiol. 2005; 46(2):170-6.

4. Rigatelli G, Zamboni A, Cardaioli P. Three-dimensional rotational digital angiography in a complicated case of patent ductus arteriosus transcatheter closure. Catheter Cardiovasc Interv. 2007;70(6):900-3.

5. Panzer J, Taeymans Y, De Wolf D. Three-dimensional rotational angiography of a patient with pulmonary atresia intact septum and coronary fistulas. Pediatr Cardiol. 2008; 29(3):686-7.

6. Wielandts JY, De Buck S, Ector J, Lagerche A, Willems R, Bosmans $\mathrm{H}$, et al. Three-dimensional cardiac rotational angiography: effective radiation dose and image quality implications. Europace. 2010;12(2):194-201. 